\title{
High-throughput micromixers based on acoustic streaming induced by surface acoustic wave
}

\author{
Trung-Dung Luong • Vinh-Nguyen Phan • \\ Nam-Trung Nguyen
}

\begin{abstract}
Flow characteristics in microfluidic devices is naturally laminar due to the small channel dimensions. Mixing based on molecular diffusion is generally poor. In this paper, we report the fabrication and characterization of active surface-acousticwave-driven micromixers which exploit the acoustic streaming effect to significantly improve the mixing efficiency. A side-by-side flow of water and fluorescent dye solution was driven by a syringe pump. Surface wave with a frequency of $13 \mathrm{MHz}$ was launched perpendicular to the flow. The wave was generated by two designs of interdigitated electrodes on $\mathrm{LiNbO}_{3}$ substrate: parallel electrodes and focusing electrodes. The mixing efficiency was observed to be proportional to the square of the applied voltage. Under the same applied voltage, the focusing type offers a better mixing efficiency. The fabri-
\end{abstract}

N. T. Nguyen

School of Mechanical and Aerospace Engineering

Nanyang Technological University

50 Nanyang Avenue, Singapore, Singapore 639798

Tel.: +65-6790-4457

Fax: +65-6792-4062

E-mail: mntnguyen@ntu.edu.sg 
cation of the micromixer is compatible to current technology such as soft lithography and deep reactive ion etching. Despite the high throughput and fast mixing time, the mixer design is simple and could be integrated into any microfluidic platform.

Keywords micromixers, surface acoustic wave, acoustic streaming, active mixer

\section{Introduction}

Mixing at microscale is a difficult task due to laminar-flow characteristics. In general, mixing in high-throughput applications require an active mixer which utilizes external disturbances. Different types of external disturbances have been reviewed [1] in micromixing such as pressure [2], electrohydrodynamics [3], dielectrophoresis [4], electroosmosis [5], magnetohydrodynamics [6], and heat [7]. Acoustic energy is another candidate for realizing active mixers. Yang et al. [8] generated an acoustic field using a piezoelectric lead zirconate titanate (PZT) membrane to enhance mixing. Bubble-based acoustic mixers $[9,10]$ have shown significant improvement in mixing performance. Exploiting surface acoustic wave (SAW) to induce acoustic streaming is another method to enhance mixing. A SAW device consists of a pair of interdigitated electrodes deposited on a piezoelectric material. Applying an alternate-current (AC) voltage on the electrodes at a right frequency will launch an elastic wave along the substrate surface. Compared to other methods, the advantages of SAW based micromixer are its simple fabrication, large disturbance force and especially fast operation.

In the literature, SAW-based micromixer have been categorized under SAW-driven mixer in both droplet-based and continuous-flow microfluidics. In droplet-based micromixer, SAW has been demonstrated as a driving and disturbance source for rapid mixing [11-14]. The working principle of SAW-driven droplet-based micromixer relies 
on strong internal flow within the droplet and induced by SAW. Mixing quality is reported to be effective for droplet with diameters larger than the SAW wavelength due to better coupling effect of the acoustic energy into the droplet. By tuning the SAW input power, mixing rate and mixing efficiency could be adjusted accordingly. However, very few studies on SAW-based micomixer for continuous-flow microfluidics have been reported in the literature. Srithiran et al [15] demonstrated a SAW mixer in a Y-shaped channel. To maximize the acoustic effect, the SAW device is placed directly under the junction of the two inlets. The wave propagation is designed to be perpendicular to the flow direction. Different voltages were used to verify the mixing efficiency at a fixed flow rate of $250 \mu \mathrm{m} / \mathrm{s}$. Complete mixing was achieved at a distance of $2 \mathrm{~mm}$ downstream. Tseng et al. [16] also reported a micromixer utilizing SAW on a Y-shaped channels. Two designs were employed: the parallel type and the transversal type. In these designs, the wave propagation is parallel and perpendicular to the flow direction, respectively. The SAW is placed away from the channel. This paper reported a mixing efficiency of more than $80 \%$ at $2 \mathrm{~mm}$ downstream from the inlet junction with a Peclet number on the order of $\mathrm{Pe}=7 \times 10^{3}$.

In this paper, the concept of SAW induced mixing of continuous flow is applied to a micromixer working with a high sample flow rate. Relatively high flow rates of $2 \mathrm{ml} / \mathrm{h}$, $5 \mathrm{ml} / \mathrm{h}$ and $10 \mathrm{ml} / \mathrm{h}$ were investigated. Water and fluorescent dye solution were used in a side-by-side configuration for the mixing experiments. Interdigitated electrodes are placed from a distance perpendicular to the mixing channel. In our work, the Peclet number could be as high as $\mathrm{Pe}=74.4 \times 10^{3}$. Compared to the above mentioned two SAW-based micromixers $[15,16]$, our Peclet number is much higher . Good mixing was achieved at a distance of $10 \mathrm{~mm}$ downstream from the inlet even at such high Peclet number. For the first time, we employed focusing interdigitated electrodes to 
concentrate the acoustic energy and therefore significantly improved the mixing quality compared to the traditional parallel interdigitated electrodes. The fabrication of our micromixers is based on standard lithography and etching processes. Following, the design, the fabrication and the characterization of these SAW-based micromixers are described.

\section{Device concept and fabrication technology}

\subsection{Acoustic streaming}

As mentioned above, the so-called surface acoustic wave is generated by interdigitated electrodes deposited on a piezoelectric substrate. The elastic wave travels along a path and its energy is confined to a thin layer at the substrate surface. Upon interacting with the liquid, leaky acoustic wave is launched into the liquid at a refraction angle $\theta_{\mathrm{R}}$ according to Snell's law:

$$
\theta_{\mathrm{R}}=\arcsin \left(\frac{V_{\mathrm{l}}}{V_{\mathrm{s}}}\right)
$$

where $V_{\mathrm{l}}$ and $V_{\mathrm{s}}$ are the propagation velocities of the wave in the liquid and the substrate, respectively. Acoustic streaming is induced, as the solid substrate interacts with the liquid. Since the wave is launched perpendicularly to the flow direction, acoustic streaming improves transversal mass transport and rapidly mixes the liquids, Figure 1. Since acoustic streaming is a non-linear, second-order phenomenon, the velocity of acoustic streaming is proportional to the square of the applied voltage [17]. Mixing efficiency is therefore expected to be a second-order function of the applied voltage. 
2.2 Device concept

Our SAW-driven micromixer consists of a microchannel bonded to a piezoelectric substrate. The microchannel has two inlets and two outlets. The detailed dimensions of the mixing channels are depicted in Fig. 2. The liquids to be mixed are introduced in a side-by-side configuration. The electrodes of the conventional parallel design and the focusing design are shown in Fig. 2. When an radio frequency (RF) signal is applied to the electrode, SAW is generated and propagates transversal to the mixing channel. Acoustic streaming occurs when the wave interfere the liquids. Mixing is induced due to the disturbance of the transversal acoustic streaming. By varying the applied voltage and the flow rate, mixing effects can be studied. The electrodes of the focusing design are concentric arcs with an opening angle of $90^{\circ}$. By adapting the arc-shaped interdigitated electrode design, the acoustic energy can be focused [18]. The propagation and distribution of acoustic energy was described using Gaussian beam theory elsewhere [19]. The focused wave energy increases the amplitude of the wave and generates a larger disturbance force as compared to the traditional parallel design. The channel is positioned at the focal point to optimally exploit the effect for mixing purpose.

\subsection{Fabrication technology}

The first step in the fabrication process of the micromixer was the preparation of the SAW substrate as shown in Figure 3(a). A $128^{\circ}$ rotated Y-cut $\mathrm{LiNbO}_{3}$ substrate of $500 \mu \mathrm{m}$ thickness was patterned with the positive photoresist AZ9260 (AZ Electronics Material) using photo lithography. The wafer was then cleaned by a mild oxygen plasma (Technics). Subsequently, a $30 \mathrm{~nm}$ titanium (Ti) and $300 \mathrm{~nm}$ gold (Au) were sputtered by a RF-magnetron system (Coxial Technology) on the patterned substrate. 
The electrodes were released by lift-off of the photoresist layer. The second step is the fabrication of the mixing channel in polydimethylsiloxane (PDMS). The mixing channel has a width of $400 \mu \mathrm{m}$ and a height $50 \mu \mathrm{m}$. A silicon substrate was patterned with positive photoresist AZ9260 and deep-etched by deep reactive ion etching (DRIE)(Surface Technology System) to form the silicon mould. The depth of the pattern defines the channel height of $50 \mu \mathrm{m}$. PDMS (Dow Corning) was mixed in a ratio of 10 to 1 and degassed for 30 minutes using a vacuum chamber. The PDMS was subsequently casted on the silicon mold, cured at $80^{\circ} \mathrm{C}$ for one hour, and then peeled off as shown in Fig. 3(b). Fluidic interconnects were punched through the PDMS part by a puncher (Harris Unicore, Jed Pella, Inc). The last step is the bonding process of the piezoelectric substrate to the PDMS piece with the mixing channel. Both the piezoelectric substrate and the PDMS piece were first treated with oxygen plasma, aligned and brought in contact for bonding. A post-bake process at $80^{\circ} \mathrm{C}$ for 30 minutes was required to promote a stronger bonding strength.

\section{Experimental setup}

The interdigitated electrodes have ten fingers pair with and electrode width and a spacing of $125 \mu \mathrm{m}$ each. The device was characterized using a RF network analyzer (Agilent E5061A) using the single electrode pair as both the emitter and receiver. The first resonance frequency of the generated SAW was measured at $13 \mathrm{MHz}$. A continuous AC signal was generated by a signal generator (Tektronix AFG3022) and amplified by a RF power amplifier (EN440LA, $35 \mathrm{~W}$ ). The signal was subsequently fed directly to the interdigitated electrodes to generate the SAW. 
The liquids are delivered from two $2.5 \mathrm{ml}$ glass syringes via plastic tubing (ColePalmer). Both syringes were driven by a syringe pump (Cole-Parmer, 749000-05). The first liquid used in our experiment is DI water from MiliQ system (Milipore). The second liquid is a solution of a green fluorescent dye (fluorescein disodium salt $\mathrm{C}_{20} \mathrm{H}_{10} \mathrm{Na}_{2} \mathrm{O}_{5}$, Acid Yellow 73) and DI water. The fluorescent dye has an excitation wavelength of $540 \mathrm{~nm}$ and emission wavelength of $610 \mathrm{~nm}$. The mixing process was observed with the help of an inverted microscope (Nikon Eclipse TE2000-S) using an excitation filter for $540 \mathrm{~nm}$, a dichroic mirror for $565 \mathrm{~nm}$ and an emission filter for $605 \mathrm{~nm}$. The images were captured by a high-speed monochrome charge couple device (CCD) camera (Photron Fastcam APX RS). The recording speed used in the experiment was 50 frames per second. The mixing quality was evaluated based on the intensity value of images captured which are proportional to the actual concentration of the fluorescent dye. The images were taken at the end of the channel which is $10 \mathrm{~mm}$ downstream from the inlet of the mixing channel.

The fluorescent images of the mixing channel were recorded before and after launching the SAW. To quantitatively characterize the mixing effectiveness of our mixer, a region of interest (ROI) was defined in the captured images and processed by a customized MATLAB program. Intensity values of each pixel were first captured. The average intensity of each line $i$ along the width of ROI at time $t$ was subsequently extracted as $I_{i}(t)$. This value is then normalized based on the maximum value $I_{\max }$ and the minimum value $I_{\min }$ of the unmixed states:

$$
\overline{I_{i}}(t)=\frac{I_{i}(t)-I_{\min }}{I_{\max }-I_{\min }}
$$


where $\overline{I_{i}}(t)$ is the normalized average intensity of line $i$ at time $t$. The mixing efficiency at time $t$ is calculated as:

$$
\eta(t)=1-\frac{\sqrt{\frac{1}{N} \sum_{i=1}^{N}\left(\overline{I_{i}}(t)-\overline{I_{\infty}}\right)^{2}}}{\sqrt{\frac{1}{N} \sum_{i=1}^{N}\left(\overline{I_{i}}(0)-\overline{I_{\infty}}\right)^{2}}}
$$

where $N$ is the number of lines along the width of ROI, $\overline{I_{i}}(0)$ and $\overline{I_{i}}(t)$ are the normalized average intensity value of line $i$ before turning on the SAW $(t \leq 0)$ and time $t$. The normalized intensity value of each line in the perfectly mixed state is denoted as $\overline{I_{\infty}}$. Due to the same flow rates of the liquids at the inlets, this value is $\overline{I_{\infty}}=0.5$ in our case. The key parameters governing the flow characteristics and the mass transport are the Reynolds number Re and the Peclet number Pe. The Reynolds number is the ratio between the advective transport and momentum transport:

$$
\operatorname{Re}=\frac{U D_{\mathrm{h}}}{\nu}
$$

where $U$ is the mean flow velocity, $D_{\mathrm{h}}$ is the hydraulic diameter of the channel and $\nu$ is the kinematic viscosity of the fluid $\left(\nu=0.8 \times 10^{-6} \mathrm{~m}^{2} / \mathrm{s}\right)$. The Peclet number Pe is the ratio between the advective transport and the diffusive transport:

$$
\mathrm{Pe}=\frac{U W}{D}
$$

where $W$ is the width of the channel, and $D$ is the diffusion coefficient of the fluorescent dye. The diffusion coefficient of the fluorescent dye used in our experiments is $D=$ $1.5 \times 10^{-9} \mathrm{~m}^{2} / \mathrm{s}$. In this study, mixing experiments at three different flow rates of $2 \mathrm{ml} / \mathrm{h}, 5 \mathrm{ml} / \mathrm{h}$ and $10 \mathrm{ml} / \mathrm{h}$ were carried out. Table 1 summarizes the corresponding Reynolds numbers and Peclet numbers used in our experiments. 
Table 1 Flow rates, Reynolds numbers and Peclet numbers used in the experiment.

\begin{tabular}{lll}
\hline Flow rate $(\mathrm{ml} / \mathrm{h})$ & Reynolds number & Peclet number \\
\hline 2 & 3.1 & $14.8 \times 10^{3}$ \\
5 & 7.7 & $37.2 \times 10^{3}$ \\
10 & 15.4 & $74.4 \times 10^{3}$ \\
\hline
\end{tabular}

\section{Results and discussion}

The mixing effect due to acoustic disturbance was recorded by the CCD camera. Representative intensity images of the channel end at the beginning of the capturing process $(t \leq 0 \mathrm{~s})$ and at $t=0 \mathrm{~s}$ after the capturing trigger are shown in Fig. 4. Normalized intensity values and the mixing efficiencies of the images in Fig. 4 are shown in Figure 5. Without acoustic disturbance, mixing in the channel solely relies on molecular diffusion. The recorded images (Fig. 4) and the evaluated data (Fig. 5) show that mixing at $t \leq 0 \mathrm{~s}$ is very poor and the efficiency is close to 0 . When a driving voltage of an peakto-peak amplitude of $33 \mathrm{~V}$ was applied to the electrodes, transversal acoustic streaming occurred and induced mixing can be observed immediately. The mixing process starts at $t>0 \mathrm{~s}$ and mixing efficiency increases with time as shown in Fig. 5.

A remarkable improvement in mixing efficiency was observed with the focusing design. The mixing efficiency was 0.78 while the mixing efficiency of the parallel design remained at about 0.5 . At the same applied voltage, focusing design offers a much better mixing quality compared to the parallel design. It is understandable by that fact that focusing SAW delivers a much larger disturbance energy to a confined area in the mixing channel (Fig. 3(c)) compared to a weaker energy spread over the entire channel in the case of parallel SAW (Fig. 3(b)). The stronger the disturbance energy, the better is the mixing quality. 
Mixing quantification could also be evaluated through the probability distribution function (PDF) or the histogram of the concentration of the images [20]. The probability values are obtained through the normalized intensity value of the images. These values are shown in Fig. 6 under an applied peak-to-peak voltage of 80 V. The Peclet number in this case is $74.4 \times 10^{3}$, higher than that of Figs. 4 and 5. For parallel design, two peaks are observed near each side of the intensity spectrum indicating poor mixing,

Fig. 6(b). Good mixing achieved by focusing SAW is demonstrated as a sharp peak at the center of the PDF, Fig. 6(a).

Figure 7 shows the mixing efficiency at different Peclet numbers driven by different voltages measured at the channel end. The results clearly show that under a lower applied voltage and at the same flow rate focusing SAW improves mixing efficiency significantly compared to parallel SAW . With a peak-to-peak applied voltage of $80 \mathrm{~V}$, mixing efficiency of $\eta=0.88$ for a Peclet number as high as $\mathrm{Pe}=74.4 \times 10^{3}$ was achieved. Even with the applied peak-to-peak voltage of $100 \mathrm{~V}$, the mixing efficiency of the parallel design at a lower Peclet of $\mathrm{Pe}=14.8 \times 10^{3}$ was only about $\eta=0.78$.

The mixing efficiency versus voltage curve has two distinct features. The first feature is its the parabolic shape at lower voltages. This feature implies that mixing efficiency is a second-order function of the applied voltage. This feature is in agreement with the fact that mixing efficiency is proportional to the velocity of acoustic streaming. Acoustic streaming velocity in turn is a second-order function of the applied voltage as reported by Nguyen et al. [17]. The second feature is the limiting value for the mixing efficiency at different flow rates. Since the focusing design can achieve better mixing at lower applied voltage, the limiting value is observed to be 0.9 as shown in Fig. 7(b). Further increase of the applied voltage will not improve the mixing efficiency. The efficiency tends to converge to this limiting value faster at lower Peclet 
numbers. Based on the trend shown in Fig. 4(a), the parallel design could reach this limiting value at a higher voltage beyond $100 \mathrm{~V}$. The effect of Peclet number on the efficiency curve is similar to that of a focused design.

The focused SAW design confines the wave to a small area and improves mixing. However, a high applied voltage could also result in heating of the substrate and the samples inside the microchannel. Heating effect could pose a problem for temperature sensitive samples. An infrared (IR) camera (NEC Thermal Tracer TH9100MR) was used to investigate the thermal effect of the focusing SAW device. Fig. 8(a) shows the measured temperature distribution on the substrate under an applied voltage of $75 \mathrm{~V}$. The highest temperature of $73{ }^{\circ} \mathrm{C}$ was observed in the area matching with the focal point of the IDT. The relationship between the measured temperatures and applied voltages is shown in Fig. 8(b). The induced temperature increase is not necessarily a drawback. This phenomenon can be useful for applications requiring controlled heating such polymerase chain reaction.

One design factor that could affect mixing efficiency is the thickness of the side channel of the PDMS cover. The thicker the side wall is, the more acoustic energy is absorbed hence the lower the mixing efficiency will be. The thickness of the PDMS side wall in the above experiments was $1.5 \mathrm{~mm}$, Fig. 3(a). To verify the above hypothesis, we carried out an experiment for parallel design (at $100 \mathrm{~V}$ and $\mathrm{Pe}=74.4 \times 10^{3}$ ) on a device with $6 \mathrm{~mm}$ wall thickness. The mixing efficiency in this case was 0.15 dropping by $50 \%$ as compared to 0.3 (achieved with a $1.5-\mathrm{mm}$ wall thickness). To minimize wave damping, the side channel should be optimized to be as thin as possible but still maintains adequate bonding and sealing integrity. 


\section{Conclusion}

This paper reports the design, fabrication and characterization of active micromixers based on acoustic streaming induced by SAW. Gold interdigitated electrode was deposited on a $\mathrm{LiNbO}_{3}$ by a lift-off process. The PDMS piece with the mixing channel was fabricated using a silicon mould. The channel and the substrate were bonded by a simple oxygen plasma process. Focusing and parallel designs of the interdigitated electrodes were used to generate the surface acoustic wave. A side-by-side flow configuration with water and fluorescent dye solution was used in our experiment. The transversal acoustic wave interacts with the liquids and induces rapid mixing. Mixing quality is significantly enhanced compared to the diffusive mixing, especially at the high-Peclet-number condition of our experiments. The focusing design shows a superior performance compared to the parallel design. The mixing speed of our device is fast. Good mixing could be achieved within few tenths of a millisecond. The device provides a mixing efficiency close to 0.9 under an applied peak-to-peak voltage of $80 \mathrm{~V}$ and at Peclet number as high as $74.4 \times 10^{3}$. The high Peclet number and the corresponding high flow rates make high-throughput applications with our micromixers possible. These ultrafast and high-performance micromixers could work as stand-alone devices or could be integrated into a more complex microfluidic platform.

\section{Acknowledgement}

The authors would like to thank Prof. Claus-Dieter Ohl from School of Physical and Mathematical Sciences, Nanyang Technological University for the use of his power amplifier. 


\section{References}

1. Nguyen NT, Wu Z (2005) Micromixers - A review. Journal of Micromechanics and Microengineering $15(2)$.

2. Deshmukh A, Liepmann D, Pisano A (2000) Continuous micromixer with pulsatile micropumps. In: Technical Digest of the IEEE Solid State Sensor and Actuator Workshop. Hilton Head Island, SC. p 73-76.

3. Deshmukh A , Liepmann D and Pisano AP (2000) Continuous micromixer with pulsatile micropumps Technical Digest of the IEEE Solid State Sensor and Actuator Workshop (Hilton Head Island, SC):73-6.

4. Ould El Moctar A, Aubry N, Batton J (2003) Electro-hydrodynamic micro-fluidic mixer. Lab on a Chip - Miniaturisation for Chemistry and Biology 3(4): 273-280.

5. Oddy MH, Santiago JG, Mikkelsen JC (2001) Electrokinetic instability micromixing. Analytical Chemistry 73(24): 5822-5832.

6. Bau HH, Zhong J, Yi M (2001) A minute magneto hydro dynamic (MHD) mixer. Sensors and Actuators, B: Chemical 79(2-3): 207-215.

7. Mao H, Yang T, Cremer PS (2002) A microfluidic device with a linear temperature gradient for parallel and combinatorial measurements. Journal of the American Chemical Society 124(16): 4432-4435.

8. Yang Z, Matsumoto S, Goto H, Matsumoto M, Maeda R (2001) Ultrasonic micromixer for microfluidic systems. Sensors and Actuators, A: Physical 93(3): 266-272.

9. Liu RH, Yang J, Pindera MZ, Athavale M, Grodzinski P (2002) Bubble-induced acoustic micromixing. Lab on a Chip - Miniaturisation for Chemistry and Biology 2(3): 151-157.

10. Ahmed D, Mao X, Shi J, Juluri BK, Huang TJ (2009) A millisecond micromixer via singlebubble-based acoustic streaming. Lab on a Chip - Miniaturisation for Chemistry and Biology 9(18): 2738-2741.

11. Frommelt T, Kostur M, Wenzel-Schfer M, Talkner P, Hnggi P, Wixforth A (2008) Microfluidic mixing via acoustically driven chaotic advection. Physical Review Letters 100(3).

12. Wixforth A (2006) Acoustically Driven Programmable Microfluidics for Biological and Chemical Applications. JALA - Journal of the Association for Laboratory Automation 11(6): 399-405. 
13. Shilton R, Tan MK, Yeo LY, Friend JR (2008) Particle concentration and mixing in microdrops driven by focused surface acoustic waves. Journal of Applied Physics 104(1).

14. Fu YQ, Du XY, Luo JK, Flewitt AJ, Milne WI, Lee DS, Park NM, Maeng S, Kim SH, Choi YJ, Park J SAW streaming in ZnO surface acoustic wave micromixer and micropump. In., Atlanta, GA. 2007. 6th IEEE Conference on SENSORS, IEEE SENSORS 2007. p 478-483.

15. Sritharan K, Strobl CJ, Schneider MF, Wixforth A, Guttenberg Z (2006) Acoustic mixing at low Reynold's numbers. Applied Physics Letters 88(5): 1-3.

16. Tseng WK, Lin JL, Sung WC, Chen SH, Lee GB (2006) Active micro-mixers using surface acoustic waves on Y-cut 128 LiNbO3. Journal of Micromechanics and Microengineering 16(3): $539-548$.

17. Nguyen N-T, White RM (2000) Acoustic streaming in micromachined flexural plate wave devices: numerical simulation and experimental verification. IEEE Transactions on Ultrasonics, Ferroelectrics, and Frequency Control 47(6): 1463-1471.

18. Wu TT, Tang HT, Chen YY, Liu PL (2005) Analysis and design of focused interdigital transducers. IEEE Transactions on Ultrasonics, Ferroelectrics, and Frequency Control 52(8): 1384-1392.

19. Meng AH, Nguyen NT, White RM (2000) Focused flow micropump using ultrasonic flexural plate waves. Biomedical Microdevices 2(3): 169-174.

20. Nguyen NT (2009) Micromixers: Fundamentals, Design and Fabrication. William Andrew. 


\section{List of Figures}

- Figure 1: Schematic representation of the interaction between SAW and the liquid in the mixing channel.

- Figure 2: Fabrication process of the micromixers: (a)Patterning of interdigitated electrodes by lift-off process; (b) PDMS part with mixing channel fabricated with a silicon mold; (c) Bonding of the substrate and the PDMS part.

- Figure 3: Schematic of the SAW micromixer: (a) Geometry and dimension of the mixing channel (length unit is millimeter); (b) Parallel interdigitated electrodes design; (c) Focusing interdigitated electrode design.

- Figure 4: Mixing behavior at the end of the mixing channel of (a) focusing design and (b) parallel design when applied voltage is zero. Mixing state induced by SAW of (c) focused design and (d) parallel design when a RF signal of $33 \mathrm{~V}$ peak-to-peak is applied to the electrodes. Clear side-by-side interface of DI water and fluorescent solution is observed in (a) and (b). Better mixing quality is achieved by focusing SAW compared to parallel SAW. The flow directions are denoted by the white arrow. The Peclet number is $37.2 \times 10^{3}$. The boxes are the ROIs for the later evaluation of the mixing efficiency.

- Figure 5: Mixing efficiency as function of time of the ROI indicated as the box in Figure 4 (applied voltage of $33 \mathrm{~V}$ and Peclet number is $37.2 \times 10^{3}$ ) for the focusing design and the parallel design. The inserts are the corresponding normalized intensity values.

- Figure 6: Probability distribution function of a ROI for (a) the parallel design and (b) the focused design. The data are evaluated at $0.6 \mathrm{~s}$ after turning on the SAW. The applied peak-to-peak voltage is about $80 \mathrm{~V}$. The Peclet number is $74.4 \times 10^{3}$. 
The inserts show the corresponding normalized intensity values across the channel width.

- Figure 7: Mixing efficiency as function of the applied voltages at different Peclet numbers (solid lines are polynomial fits): (a) parallel design; (b) focusing design.

- Figure 8: Temperature distribution on the substrate induced by the focusing design (a) temperature of substrate captured by the IR camera at $75 \mathrm{~V}$; (b) Maximum temperature at the focal point versus applied voltage. 


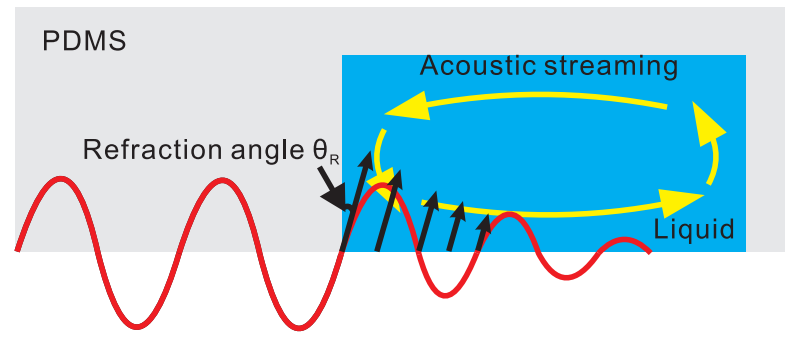

Fig. 1 Schematic representation of the interaction between SAW and the liquid in the mixing channel.

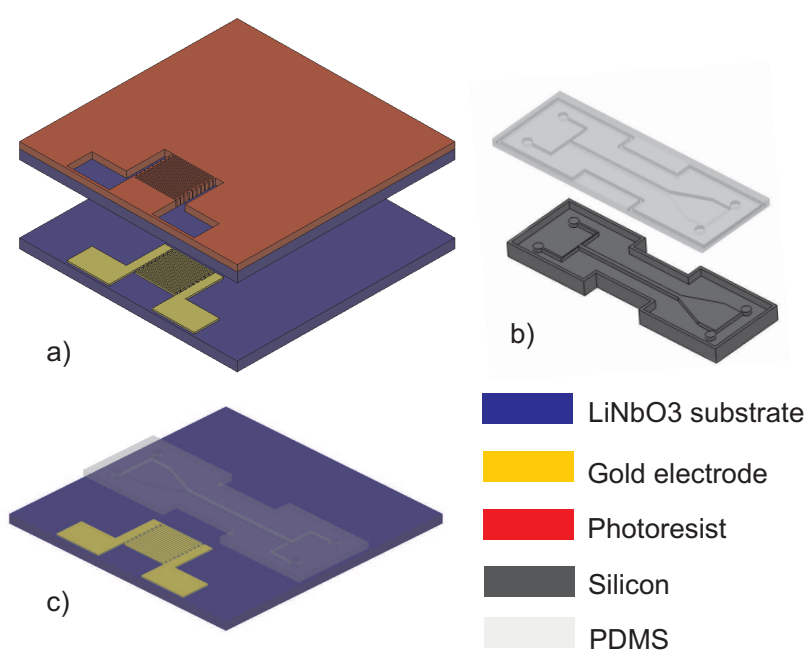

Fig. 2 Fabrication process of the micromixers: (a)Patterning of iterdigitated electrodes by liftoff process; (b) PDMS part with mixing channel fabricated with a silicon mold; (c) Bonding of the substrate and the PDMS part. 


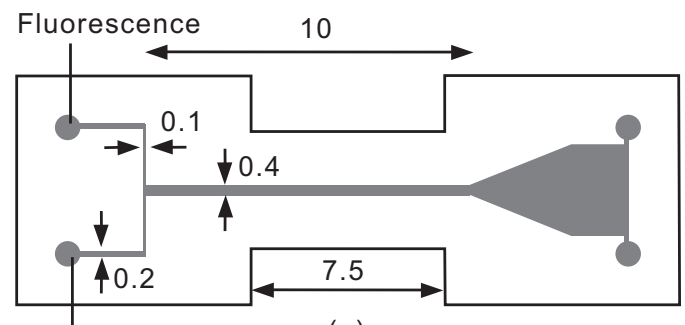

Water

(a)
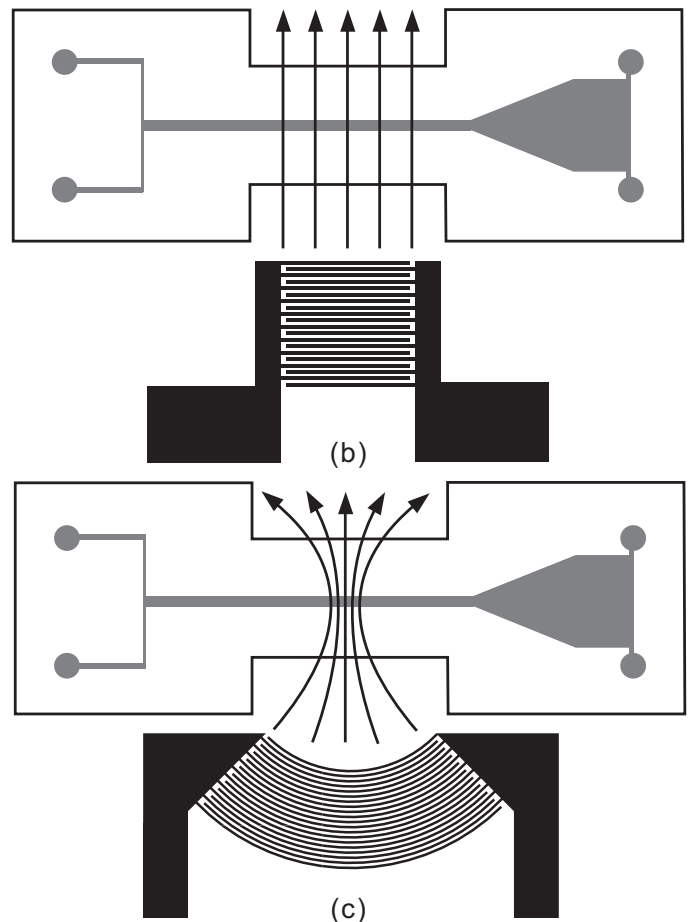

Fig. 3 Schematic of the SAW micromixer: (a) Geometry and dimension of the mixing channel (length unit is millimeter); (b) Parallel interdigitated electrodes design; (c) Focusing interdigitated electrode design. 


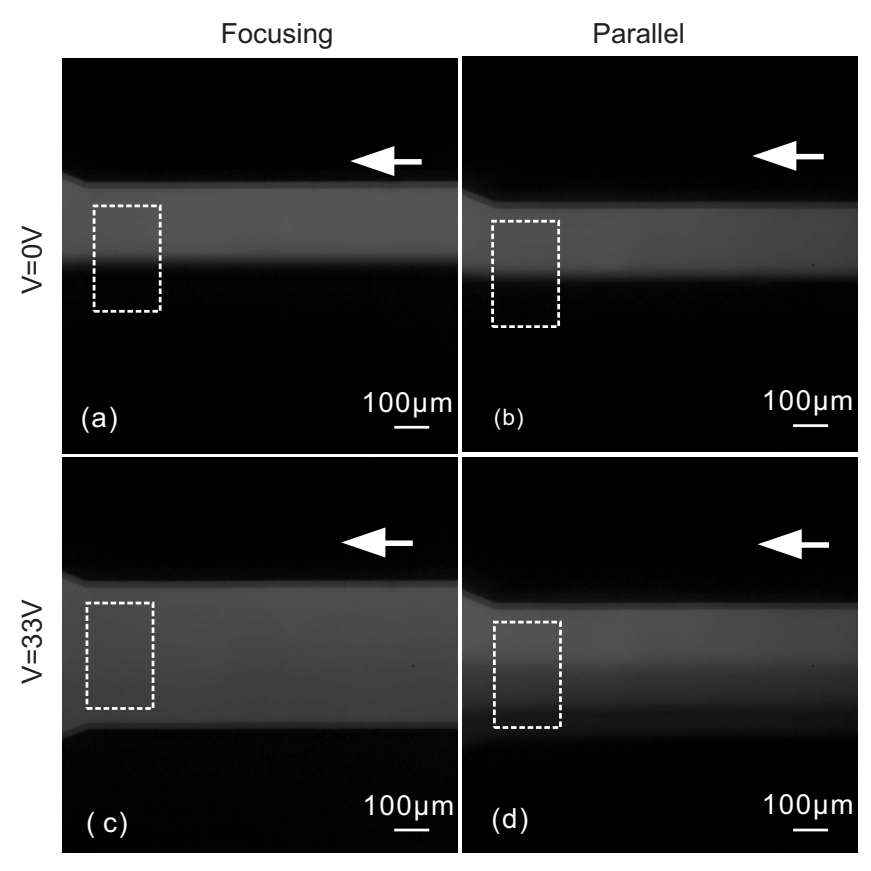

Fig. 4 Mixing behavior at the end of the mixing channel of (a) focusing design and (b) parallel design when applied voltage is zero. Mixing state induced by SAW of (c) focused design and (d) parallel design when a RF signal of $33 \mathrm{~V}$ peak-to-peak is applied to the electrodes. Clear side-by-side interface of DI water and fluorescent solution is observed in (a) and (b). Better mixing quality is achieved by focusing SAW compared to parallel SAW. The flow directions are denoted by the white arrow. The Peclet number is $37.2 \times 10^{3}$. The boxes are the ROIs for the later evaluation of the mixing efficiency. 


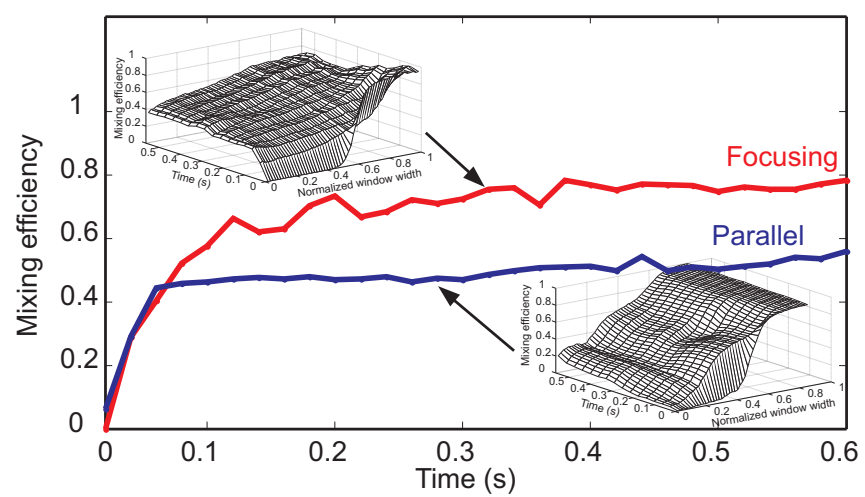

Fig. 5 Mixing efficiency as function of time of the ROI indicated as the box in Figure 4 (applied voltage of $33 \mathrm{~V}$ and Peclet number is $37.2 \times 10^{3}$ ) for the focusing design and the parallel design. The inserts are the corresponding normalized intensity values.
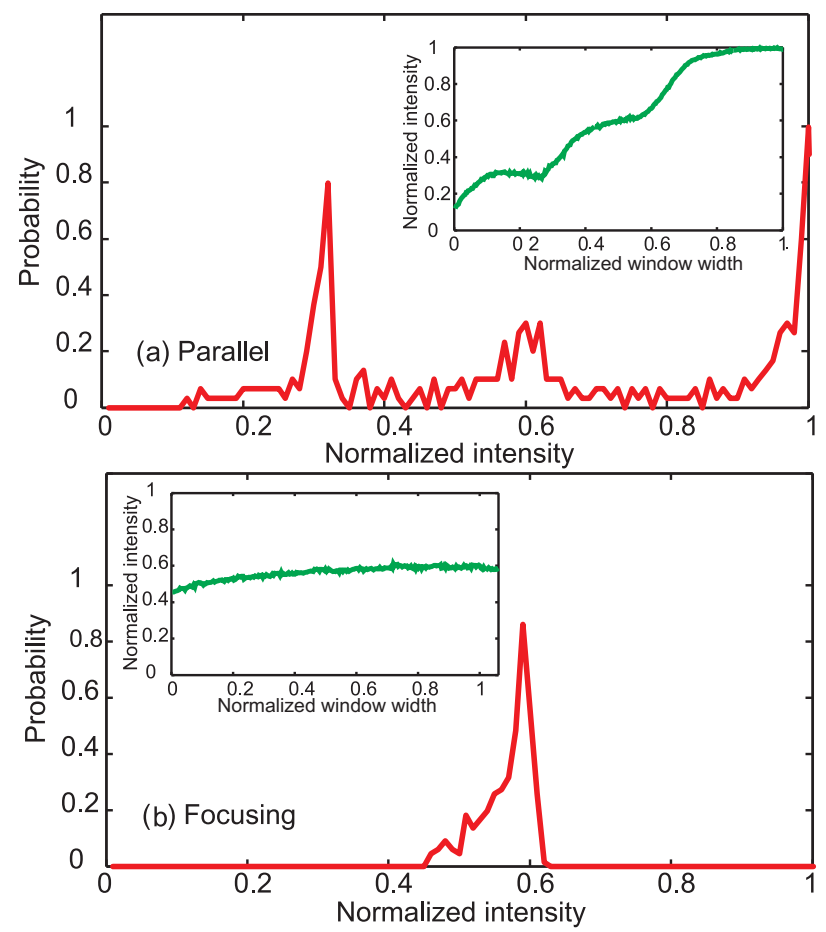

Fig. 6 Probability distribution function of a ROI for (a) the parallel design and (b) the focused design. The data are evaluated at $0.6 \mathrm{~s}$ after turning on the SAW. The applied peak-to-peak voltage is about $80 \mathrm{~V}$. The Peclet number is $74.4 \times 10^{3}$. The inserts show the corresponding normalized intensity values across the channel width. 

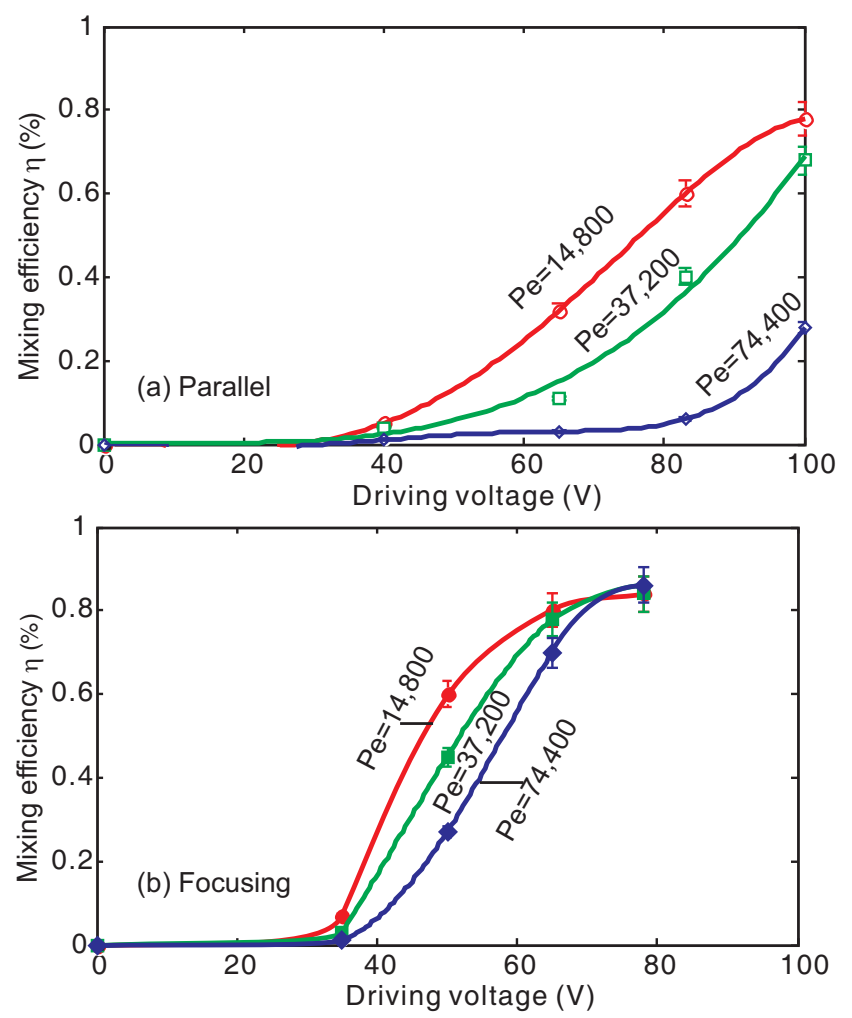

Fig. 7 Mixing efficiency as function of the applied voltages at different Peclet numbers (solid lines are polynomial fits): (a) parallel design; (b) focusing design. 


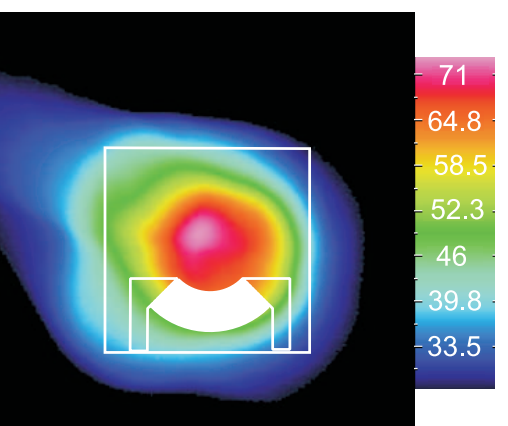

(a)

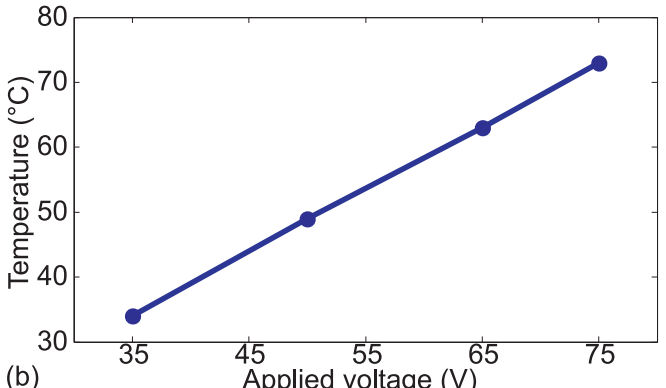

Fig. 8 Temperature distribution on the substrate induced by the focusing design (a) temperature of substrate captured by the IR camera at $75 \mathrm{~V}$; (b) Maximum temperature at the focal point versus applied voltage. 